\title{
EPIDEMIOLOGY
}

UDC 579.222.7, 614.446.33

\section{The issue of nosocomial infection caused by Klebsiella pneumoniae blaNDM-type}

O. V.Mironenko ${ }^{1,2}$, V. V.Selnitseva ${ }^{3}$, L.A.Soprun ${ }^{2}$, E. N. Shmushkevich ${ }^{3}$, A. A. Alekseev' ${ }^{1}$ A.S. Ivanov ${ }^{1}$, A. A. Tovanova ${ }^{1}$

${ }^{1}$ North-Western State Medical University named after I. I. Mechnikov, 41, Kirochnaya ul., St. Petersburg, 191015, Russian Federation

${ }^{2}$ St. Petersburg State University,

7-9, Universitetskaya nab., St. Petersburg, 199034, Russian Federation

${ }^{3}$ St. Petersburg State City Hospital no. 2,

5, Uchebny per., St. Petersburg, 194354, Russian Federation

For citation: Mironenko O. V., Selnitseva V.V., Soprun L.A., Shmushkevich E. N., Alekseev A.A., Ivanov A.S., Tovanova A.A. The issue of nosocomial infection caused by Klebsiella pneumoniae blaNDM-type. Vestnik of Saint Petersburg University. Medicine, 2020, vol. 15, issue 2, pp. 124-133.

https://doi.org/10.21638/spbu11.2020.204

The article presents information about circulating isolates Klebsiella pneumoniae in a hospital megapolis with properties of hypervirulence and simultaneous multiresistance. The resulting K.pneumonia isolates are of particular importance due to the emergence of resistance to almost all $\beta$-lactams due to the presence of carbapenemase metal- $\beta$-lactamase. Furthermore, the isolated strains producing carbapenemases possess mechanisms of resistance to a wide range of antimicrobial preparations, and the types of infectious process caused by carbapenemazo-producing enterobacteries are characterized by a high lethality level. Microbiological, biochemical, biophysical, molecular-genetic, biological, bioinformational and statistical methods of research were used in the work. A prospective method was used to identify the source of the infections. In the first stage, a microbiological study was carried out on biomaterials obtained from patients treated in a hospital in Saint Petersburg. After a microbiological study, 52 isolates of K.pneumoniae were obtained, $53.8 \%$ of isolates had a hypermucoid phenotype and $98 \%$ had carbapenemases: blaNDM type - 49 (92\%), blaNDM+OXA-48-like - 3 (8\%). Isolates with two new phenotypes have been isolated (no. 2511 and no. 2512). Isolates of no. $2512 \mathrm{LD} 50$ had $10^{\star} 2 \mathrm{BAC} / \mathrm{ml}$, and plasmids such as Incfib(Mar), Inchilb, and Incr were also found, with Incr-A plasmid emitted encoding resistance to fluoroquinolone: $\mathrm{aac}\left(6^{\prime}\right)$-Ib-cr and to $\beta$-lactam antibiotics: blaTEM-1B. The described data confirm the opinions of the researchers about the possible formation of a new "super pathogen" - instantaneously hypervirulent and plural resistant strain of K. pneumoniae. Keywords: K. pneumonia blaNDM, nosocomial infection, epidemiological process, carbapenemaza, hypervirulence.

(c) Санкт-Петербургский государственный университет, 2020 


\section{Introduction}

According to WHO data, carbapenem-resistant enterobacteria are the main causes of nosocomial infections by the beginning of 2020. Enterobacteria due to increased morbidity and mortality among hospitalized carbapeneme patients is considered the last line of treatment for Enterobacteria infections and resistance to them is a major problem for clinicians.

As is known, the group "ESKAPE" includes antibiotic-resistant pathogens (Enterococcus faecium, Staphylococcus aureus, Klebsiella pneumoniae, Acinetobacter baumannii, Pseudomonas aeruginosa and Enterober spp.), including K. pneumoniae (Santajit, Indrawattana, 2016) [1]. In the Russian Federation, as in the rest of the world, K. pneumoniae are current hospital pathogens [2-4] and cause a wide range of pathologies in humans: sepsis, bacteria, respiratory infections, infections of the urinary system, gastro-intestinal lesions, eyes, primary abscesses, etc. [5-7].

Starting in the second half of the 1990s, carbapenemase spread became a problem in several European countries, with P. aeruginosa being the most common causative agent. Less than 10 years later, in the 2000s, K. pneumoniae began an epidemic spread in Greece, containing integrated metal- $\beta$-lactamases from Verona (Verona integron-ded metallo$\beta$-lactamase - VIM), followed by carbapenemases (Klebsiella pneumoniae carbabapenemase - KPC).

Carbapenemaza is the most common species of K.pneumoniae among enterobacteria in Europe today. Currently in Greece and Italy, about 60 and $15 \%$ (respectively) of all invasive K. pneumoniae are insensitive to carbapenemes $[1 ; 8 ; 9]$. Enterobacteria remains the main cause of death and complications of major diseases among patients in hospital.

In addition to the carbapen listed above, one of the major problems of the past few years among nosocomial infections is carbapenemase from New Delhi (New Delhi metallo- $\beta$-lactamase). Metal- $\beta$ lactamases (MBL) from New Delhi spread epidemiologically to the Indian subcontinent and the Middle East, which were then introduced to Europe. The distribution of OXA-48-like enzymes in a number of European countries began in the form of individual outbreaks, and continued rapidly [10-12].

There are quite a few K.pneumoniae classifications. This article will be guided by the epidemiological principle based on the evolutionary approach of isolating classical and hypervirulent klebsiell strains [13].

The classical K.pneumoniae causes diseases in infants and the elderly, as well as in persons with reduced immunity, who have a large number of chronic pathologies and related diseases [14]. Characterized by the presence of genes of beta-lactamase (CTX-M, TEM-, OXA-48, NDM- and KPC-types), integrons of genetic determinants, which determine their antibacterial characteristics and the distribution among microflora of treatment and prevention institutions [15]. The domestic authors describe multi-stable clinical strains of Klebsiell to cephalosporins and carbapenemes, on the territory of the Russian Federation $[3 ; 5 ; 10 ; 16]$.

With regard to the second species of K.pneumoniae, these hypervirulents are capable, causing severe, with the frequency of fatal outcome 3-55\%, diseases in people with normal immunity $[9 ; 12]$. The primary excretive abscess of the liver followed by the metastasization of the agent from the site of infection to other organs and tissues is a manifestation of hypervirulent strains, first described in Asia (India) [17]. It is known that it is the genetic determinant that defines the property of hypermucoity. So hypervirulent K. pneumoniae more 
often than classical strains are characterized by a hypermucoid phenotype $[8 ; 18]$, with molecular systems of trivalent iron absorption [12;13], with a recycling system of alloantoine [19], with synthesis of smooth lipopolysaccharide and polysaccharide capsule [10;15] and with the presence of type I fimbrial [16]. Multiresistant classical K. pneumoniae syvens ST11, ST14, ST15, ST35, ST36, ST147, ST258, ST309, ST1107 and K1-K78 types [13; 20] and hypervirulent strains K. pneumoniae with ST23, ST29, ST65, ST86, ST268, ST375, ST412 and ST420 [13; 19; 20] and K1, K2, and rarely K5, K20, K54 and K57 types [13; 16; 20].

It is most often mobile genetic elements (plasmids) that are the basis of antibiotic resistance and virulence in klebsiells, as well as in other bacteria. In South Korea, the strains K1 ST23 with the epidemic gene beta-lactamase: cephalosporinase blaCTX-M-15, which have been isolated from the hypervirulent K. pneumoniae [17], have been described. K. pneumoniae multilocus systematization allows for the detection of pathogen epidemic genetic lines, their distribution in the world, and the assessment of the contribution of different genetic lines to the spread of genetic determinants of antibiotic resistance and virulence.

For example, the hypervirulent evolutionary branch is associated with the isolates K.pneumoniae of the world's most common clonal CC23 complex (ST23 and ST218 sequels). A high degree of virulence for humans and laboratory animals is noted for the ST86 and ST65 sequel types. A wide range of ABP resistance can be found in K.pneumoniae of other genetic lines: CC258, CC11, CC15, and CCC14.

In a 2013 study in China, K.pneumoniae ST15 dominated the isolates isolated in 306 hospitals, which included pan-resistant strains that caused lung infections, kidney dysfunction, and heart failure. It is noted that K.pneumoniae ST15 is widely distributed in Denmark, Hungary, Korea, Malaysia, Singapore and Taiwan. Isolates of ST11 are also common in China, while the clebsiells of the "hybrid" ST258, formed from ST11 and ST442, are widely represented in North and Latin America, as well as in some countries of Europe.

In other countries, there have also been reports of the acquisition of hypervirulent characteristics of K.pneumoniae: Argentina - strains of K1 ST23 with carbapenemase gene K1 blaKPC-2 [21], China - strains of new ST1797 with genome blaKPC-2 [19], France - strains of K2 ST86 with genome blaCTX-M-3 [17]. In 2017, the Russian Federation described a case of meningitis in the neonatal ward of Kazan, caused by a hypermucoid, rmpA-positive strain of K.pneumoniae producing unidentified beta lactamase of the extended spectrum $[10 ; 22]$. In 2017, China recorded the acquisition of pLVPK-type plasmid virulence with multiresistant hospital strains K47 K. pneumoniae ST11 [9; 22].

In 2017, China recorded the acquisition of pLVPK-type plasmid virulence with multiresistant hospital strains K47 K.pneumoniae ST11 [9; 22]. Hospital K.pneumoniae in China have multiple drug resistance of $61.4 \%, 2.0 \%$ extreme drug resistance and $1.8 \%$ resistance to all antibiotics used.

For K.pneumoniae ST11 and ST258, KPC- and NDM-type carbapenemases are often common. Due to the active study of K.pneumoniae, new sequins of isolates are discovered every year. In the large-scale study Wang et al., in 2013, seven new gene alleles of "household" Klebsiell and 24 new sequins-s were described type, in Venezuela in $2017-3$ new sequels of type have been identified, in Germany a new highly virulent ST2398 has been described. In Germany, the new ST2398 has been described. The described data confirm the opinions of the researchers about the possible formation of a new "super pathogen" - a hypervirulent and simultaneously multi-resistant strain of K.pneumoniae. 
The purpose of our research was to study the molecular-genetic properties and regularities of epidemiological propagation in a multi-functional stationary strain of K.pneumonia, which is polyresistant to antibacterial drugs and hypervirulence.

\section{Materials and methods of research}

Microbiological, biochemical, biophysical, molecular-genetic, biological, bioinformatic and statistical research methods have been used to achieve this goal. A prospective method was used to identify the source of the infections.

In the first stage, a microbiological study was carried out on biomaterials obtained from patients treated in a multidisciplinary hospital in Saint Petersburg. The following biomaterials were obtained for the study materials: BAL $14(27 \%)$, separated from the ear $1(2 \%)$, conjunctiva $3(6 \%)$, unknown 7 (13\%), sputum $15(29 \%)$, tracheal aspirate 2 $(4 \%)$, urine $9(17 \%)$, severable from the wound $1(2 \%)$.

The composition of biomaterial samples has been estimated using two methods: a real-time polymerase chain reaction (PCR-PB) and a meagenomic study analysing gene sequences to determine the quantitative content of bacteria, Percentage representation, alpha and beta diversity. Bacteria were cultivated in dense and liquid nutrient media, storing $15 \%$ glycerine at minus $70^{\circ} \mathrm{C}$. The species was identified using VITEK-2 Compact (Biomerieux, France) and MALDI-TOF Biotyper (Bruker, Germany). Forty-six cases of PCR isolated K. pneumonia patients were analysed.

Preparation of the DNA matrix for the polymerase chain reaction. Cell thermolytes were used as the matrix for PCR. The bacteriological loop of the nocturnal bacterial culture was suspended in $0.1 \mathrm{ml}$ of the lyzing solution ( $0.25 \%$ SDS, $0.05 \mathrm{M} \mathrm{Naoh}$ ) to a concentration of $108-109 \mathrm{MEC} / \mathrm{ml}$ in test tubes with a capacity of $1.5 \mathrm{ml}$ of type Eppendorf. The samples were warmed up at $98-100^{\circ} \mathrm{C}$ on a water bath or in a solid thermostat, with $0.9 \mathrm{ml}$ of deionized water added to each test tube, the samples were centrifuged at 19,000 g for 2 minutes at a temperature of $4{ }^{\circ} \mathrm{S}$. The supernatant was sampled into sterile microfabrics and used as a 1-2 um matrix for the PCR reaction.

The commercial Genejet Plasmid Miniprep Kit (Thermo Fisher Scientific, Waltham, USA) was used to extract plasmid DNA from the bacteria according to the manufacturer's instructions. Whose principle is based on the alkaline lysis of deposited bacterial cells by centrifuging in the presence of SDS with further lysate neutralization, the removal of debris with SDS with the aid of centrifuging. Adsorption of plasmid DNA on a column with a membrane based on silica - gel and its evaporation from the membrane of the column after removal of impurities by double flushing. Plasmid profiles were visualized with an electrophoresis of $0.7 \%$ agarosis gel.

In accordance with guidance no. 4.2. 1890-04 determined the sensitivity of bacteria to BPA by micro-prospecting in Muller-Hinton broth and disco by diffusion method using commercial discs in 96-lunar tablets for the following antibiotics: cefoaxim, cephepim, ceftazidim, gentamicin, Imipenem, Meropeem, trimethodium-sulfonethoxazole, thihecycline, polymyxin B, ciprofloxacin, ceftazidim-avibakts, aztreonamavibakts. The string test was used to determine the hypermucoidality of K. pneumoniae cultures $[9 ; 22]$.

Primer-specific PCR has been used to determine K. pneumoniae strains of genetic determinants of resistance to BDP, genes associated with virulence and capsule types in 
the genomes of Enterobacteriaceae bacteria. Carbapenemase genes have been detected by PCR using the Amplysens ${ }^{\oplus}$ dr KPC/OXA-48-FL(blaKPC, blaOXA-48-like) and Amplysens ${ }^{\circ}$ dr MBL-FL (blaVIM, blaIMP, blaNDM) sets (Interlabservice). Production of oligonucleotides and sequencing of DNA sequences using the Sanger method.

The data were then evaluated using statistical and epidemiological methods. Descriptive statistics included calculations of arithmetic mean and standard deviation (Mean SD), median and 1st and 3rd quartiles (Median, [Q1;Q3]), and indications of minimum and maximum values (min-max). Based on the regression coefficients for the best model, the morbidity rate (incidence rate, IRR) and its $95 \%$ confidence interval (CI) were calculated. The result was considered statistically significant when the probabilities of a first genus error $(p)$ were less than 0.05 . For $p=0.005$, the results were considered extremely significant.

\section{Results}

52 isolates of K.pneumonia have been isolated. The age group of investigated and possible sources of infection was as follows: $24-44$ years $-9 \%, 45-60$ years $-23 \%, 61-70-$ $21 \%, 71-80-20 \%$, over $81-27 \%$. (Fig. 1).

In $57 \%$ of cases, the main diagnosis is related to the pathology of the cardiovascular system, $23 \%$ to the respiratory system, $9 \%$ to the endocrine system and $7 \%$ to the urinary system. In $43.18 \%$ of the cases, the fatal outcome was achieved.

In order to identify the mechanisms of transmission of the infection, a type of intervention was established: $34 \%$ - endoscopic manipulation, $11 \%$ - cavity operation, $20 \%$ - tracheostoma (Fig. 2).

$52 \%$ of patients were transferred between different wards. Valuable in the context of the referral mechanism is the determination of the number of interventions performed per patient: the highest in 5 operations in $33 \%$ of cases, $27 \%$ (4 interventions), $20 \%$ (3), $13 \%(2), 7 \%(1)$.

In order to study the characteristics of the source of the infection, it was found that cephalosporins were prescribed in $30 \%$ of cases, fluoroquinolone was prescribed in $17 \%$ of cases, and the following antibacterial preparations were taken in $12 \%$ of cases: nitromoidazole, aminoglycosides, carbapenemes (Fig. 3).

Of these, 28 isolates $(53.8 \%)$ had a hypermucoid phenotype, while the rest had no phenotypic reaction of $24(46.2 \%)$. The specified carbapenemase types are: blaNDM type -49 (92\%), blaNDM+OXA-48-like $-3(6 \%)$, negative -1 (2\%). Most carbapenemase-producing bacteria are resistant to extended-spectrum oxyminicephalo sporins.

By analyzing the hydrolysis of carbapenems by means of matrix-associated laser desorption-ionization time of the span mass spectrometry) (MALDI-TOF MS) production of carbapenem from the isolated isolates is confirmed for several hours. Isolates of K.pneumoniae were characterized by high cross-resistance and associated resistance. In $100 \%$ of cases, isolates were resistant to Amikatin, Cephepin, Cephaperazon, Cefatoxim, Ceftazidim, and to a pair of antibacterial preparations: Ceftazidim and Avibaktam.

In $61.1 \%$ of the cases, the resulting K.Pneumoniae isolates were resistant to gentamicin and thigecyclin, with $50 \%$ being either Impenemic-resistant or indifferent. It is $55.6 \%$ resistant to Meropinium and Polymyxin. At the same time, it is $38.9 \%$ Isolates of K. Pneumoniae are absolutely indifferent to Meropinium and Tigecyclin. The frequency 


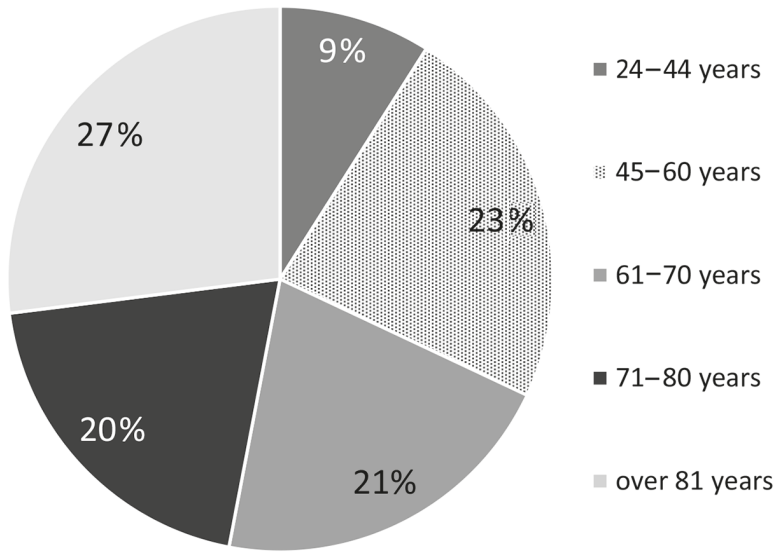

Fig. 1. Age of study group

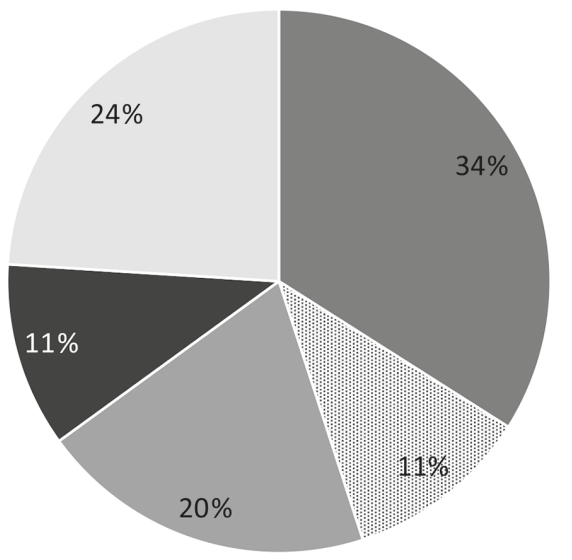

- Endoscopy

(1: Cavity surgery

- Tracheostomy

- Intubation

No

Fig. 2. Types of intervention

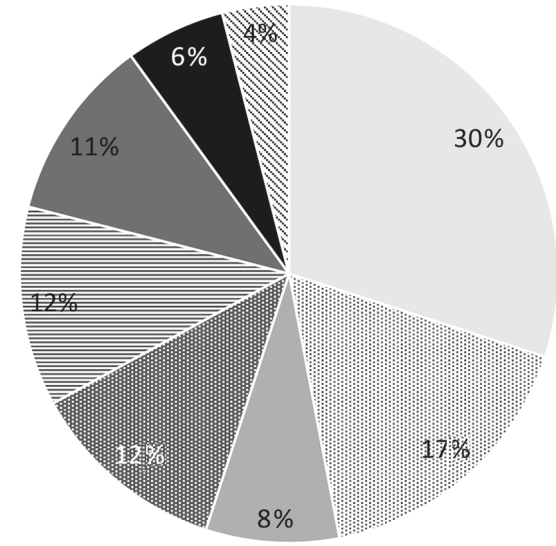

Cephalosporins

f: Fluoroquinolone

- Penicillin

Nitroimidazole

$\equiv$ Aminoglycoside

- Carbapenems

- Glycopeptides

$\approx$ No

Fig. 3. The administration of antibacterial drugs 
of stability and intermediate sensitivity to c-trimoxazole was $88.9 \%$, gentamicin $83.3 \%$, imipenem $100 \%$, meropenium $94.4 \%$, and tihecycline $100 \%$.

A relatively high sensitivity level was maintained for polymyxin B (44.4\%) and a combination of aztreonam and avibaktam $(66.7 \%)$. The best results for antibacterial therapy were found in the pair Aztreonam and Avibaktam, but even to them resistance is observed in $11.1 \%$ of cases (Table).

Table. Minimum inhibiting concentrations (MIC) of K.pneumoniae

\begin{tabular}{|l|c|c|c|c|c|}
\hline \multicolumn{1}{|c|}{ Antibiotic name } & Breakpoints & $\% \mathrm{R}$ & $\% \mathrm{I}$ & $\% \mathrm{~S}$ & MIC Range \\
\hline Amikacin & $\mathrm{S}<=8 \mathrm{R}>=32$ & 100 & 0 & 0 & $64-128$ \\
\hline Cefepime & $\mathrm{S}<=1 \mathrm{R}>=8$ & 100 & 0 & 0 & $64-512$ \\
\hline Cefoperazone & $\mathrm{S}<=1 \mathrm{R}>=4$ & 100 & 0 & 0 & $0.256-512$ \\
\hline Cefotaxime & $\mathrm{S}<=1 \mathrm{R}>=4$ & 100 & 0 & 0 & $64-512$ \\
\hline Ceftazidime & $\mathrm{S}<=1 \mathrm{R}>=8$ & 100 & 0 & 0 & $256-512$ \\
\hline Ciprofloxacin & $\mathrm{S}<=.25 \mathrm{R}>=1$ & 100 & 0 & 0 & $64-512$ \\
\hline Gentamicin & $\mathrm{S}<=2 \mathrm{R}>=8$ & 61.1 & 22.2 & 16.7 & $0.25-512$ \\
\hline Imipenem & $\mathrm{S}<=2 \mathrm{R}>=8$ & 50 & 50 & 0 & $2-256$ \\
\hline Meropenem & $\mathrm{S}<=2 \mathrm{R}>=16$ & 55.6 & 38.9 & 5.6 & $2-64$ \\
\hline Polymixin B & $\mathrm{S}<=2 \mathrm{R}>=4$ & 55.6 & 0 & 44.4 & $0.25-256$ \\
\hline Tigecycline & $\mathrm{S}<=0.5 \mathrm{R}>=1$ & 61.1 & 38.9 & 0 & $0.5-32$ \\
\hline Trimethoprim/Sulfamethoxazole & $\mathrm{S}<=2 \mathrm{R}>=8$ & 88.9 & 0 & 11.1 & $0.5-256$ \\
\hline Ceftazidime/Avibactam & $\mathrm{S}<=8 \mathrm{R}>=16$ & 100 & 0 & 0 & $256-256$ \\
\hline Aztreonam/Avibactam & $\mathrm{S}<=1 \mathrm{R}>=8$ & 11.1 & 22.2 & 66.7 & $0.125-128$ \\
\hline
\end{tabular}

After a microbiological study, 52 isolates of K.pneumoniae were obtained, $53.8 \%$ of isolates had a hypermucoid phenotype and $98 \%$ had carbapenemases: blaNDM type 49 (92\%), blaNDM+OXA-48-like - 3 (8\%) (Fig. 4). Isolates with two new phenotypes

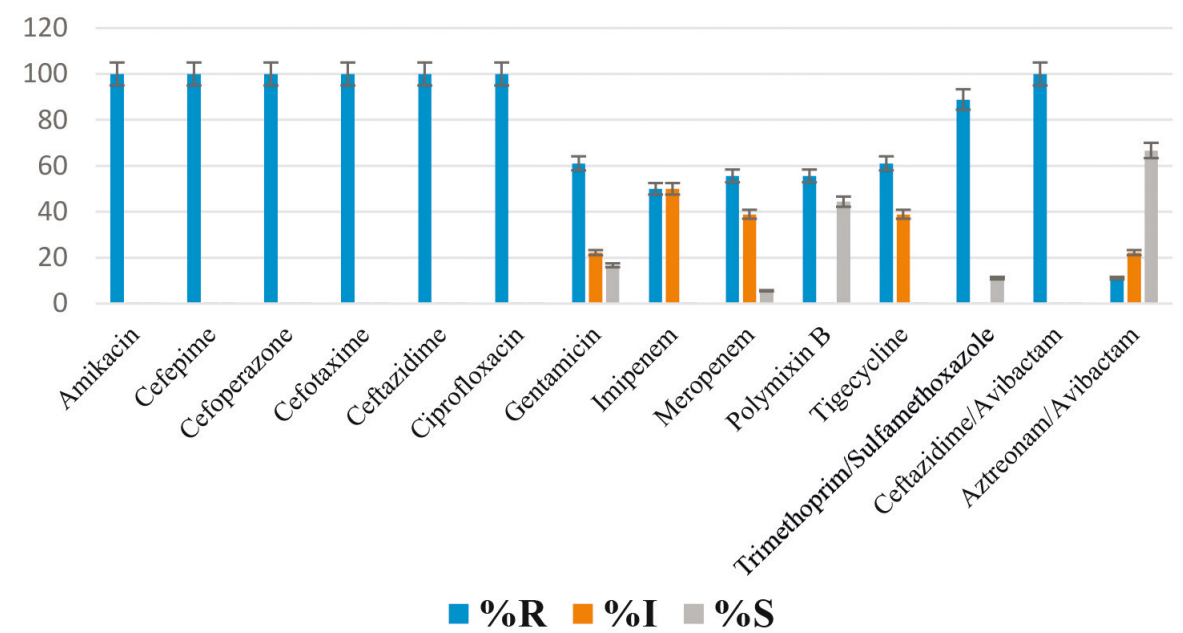

Fig. 4. Minimum inhibiting concentrations of K.pneumoniae 
have been isolated (no. 2511 and no. 2512). The isolates of $2512 \mathrm{LD} 50$ are $10^{\star} 2 \mathrm{BAC} / \mathrm{ml}$. Plasmids Incfib(Mar), Inchilb, Incr were found in isolates 2512. Secreted plasmid Incr in strain 2512 encoded resistance to fluoroquinolone: aac(6')-Ib-cr and to $\beta$-lactam antibiotics: blaTEM-1B.

\section{Discussion}

Thus, the K. pneumoniae isolates produced are of particular importance, as almost all $\beta$-lactam resistance occurs due to carbapenemase metal- $\beta$-lactamase. Furthermore, the isolated strains producing carbapenemases possess mechanisms of resistance to a wide range of antimicrobial preparations, and the infections caused by carbapenemazo-producing enterobacteria have a high lethality level.

Of particular concern is this type of metal- $\beta$-lactamase, carbapenemase, as it can cause resistance to almost all $\beta$-lactams. Furthermore, the strains producing carbapenemases often have mechanisms of resistance to a wide range of antimicrobial preparations, and the infections caused by carbapenemazo-producing enterobacteria are characterized by a high lethality level.

Until recently, the propagation of plasmid-encoded MBL was a minor threat in most geographical regions, even in countries with sporadic enzyme outbreaks, such as VIM-1 in Greece, IMP-8 in Taiwan, and IMP-1 in Japan. However, Mbls became more threatening after the NDM-1 zinc-containing carbapenemase was identified in 2009 from an isolate originating from New Delhi, India. As opposite to other MBL systems, NDM-1 quickly spread globally, being the predominant carbapenemase in the Indian subcontinent. Of great importance is the prevalence of the blaNDM gene, which has been identified in water samples and in the environment in India.

The results indicate that K.pneumoniae is a strain that is polyresistant to the antibacterial preparations used and encoded with plasmids by Incfib(Mar), Inchilb and Incr in the K.pneumonia strain. Incr's isolated plasmid encodes resistance to fluoroquinolone: aac (6')-Ib-cr and to $\beta$-lactam antibiotics: blaTEM-1B.

At present, a-lactam agent that inhibits a wide range of pathogens, including nonfergent and anaerobic pathogens has yet to be identified. This is partly due to an increase in the number of microorganisms with multiple drug stability producing Mbls and / or OXA-48 carbapenemases in combination with lactamases of different classes and the lack of an effective inhibitor of all enzymes.

Thus, it is possible that MBL, rather than serine-lactamases, may become the predominant carbapenemase in bacteria with multiple drug resistance. It is also possible that lactamase-resistant agents, such as cephiderocol (S-649266), or lactam-free agents, such as plazomycin, omadacyclin, or eraavacycline, provide reliable and safe therapeutic alternatives against the most carbapen-resistant microorganisms.

In conclusion, it should be noted that lactamases, which are among the "oldest enzymes", have proved to be the most studied with one side and with the other the most problematic determinants of resistance to antibiotics. The fact that genes encoding many lactamases are "travelling" together on movable elements - plasmids, determines the possibility of transmission of resistance factors for other classes of antibiotics. Additional unique lactamases with unusual properties can be expected to be identified due to the 
widespread distribution of these genes both in the environment and in the hospital environment due to the extensive use and use of antibiotics.

Therefore, the identification and subsequent monitoring of microbial agents in each health facility should be more demanding, with a description of each sub-unit on the circulating microbial unit. This will help to provide a scientific basis for the development of new antimicrobial agents that will help to avoid new outbreaks of infections.

\section{Conclusion}

The findings of the study indicate the need for continuous epidemiological surveillance of the circulation of strains in multi-hospital facilities in order to prevent nosocomial infections. There is also a need to develop timely tactics for effective antibiotic therapy and adequate preventive measures.

\section{References}

1. Reshed'ko G.K., Rjabkova E.L., Krechikova O.I., Sukhorukova M.V., Shevchenko O.V., Edelstain M. V., Kozlov R.S., Turkutjukov V.B., Nekhaeva G. I., Bochkarev D. N., Rozanova S. M., Boronina L.G., Agapova E.D., Marusina N.E., Multikh I.G., Taraban V.K., Zdzitovetsky D.E., Sarmatova N.I., Tikhonov Yu. G., Polikarpova S. V., Bolschakov L. V., Bogomolova N.S., Dmitrieva N. V., Petuchova I.N., Galeeva E.V., Kruglov A.N., Vischelesskaya N.D., Aleksandrova I.A., Beloborodova N. V., Vostrikova T. Yu., Iljina V.N., Ivanova S. F., Skalsky S. V., Zubareva N. A., Suborova T. N., Malischeva V.A., Osipova V. V., Shchetinin E.V., Nikolaeva T. A., Martianova N. M., Gudkova L. V., Ortenberg O.A., Uschakova M.A., Khasanova S. G., Gabbasova L.A., Kolesnik T. I., Toropova I. A., Paljutin Sch.H., Monachova S.I. Antimicrobial Resistance Patterns of Gramnegative Nosocomial Pathogens in Russian ICUs. Klinicheskaia mikrobiologiia i antimikrobnaia khimioterapiia, 2008, vol. 10, no. 2, pp. 96-112. (In Russian)

2. European Committee on Antimicrobial Susceptibility Testing. EUCAST guidelines for detection of resistance mechanisms and specific resistances of clinical and/or epidemiological importance, version 1.0, 2012. Available at: https://www.eucast.org/resistance_mechanisms (accessed: 27.09.2020).

3. Santajit S., Indrawattana N. Mechanisms of Antimicrobial Resistance in ESKAPE Pathogens. BioMed Res. Int., 2016, vol. 2016, Article ID 2475067. https://doi.org/10.1155/2016/2475067.

4. Rosenthal V.D., Al-Abdely H.M., El-Kholy A. A., et al. International Nosocomial Infection Control Consortium report, data summary of 50 countries for 2010-2015: Device-associated module. Am. J. Infect. Control, 2016, vol. 44, no. 12, pp. 1495-1504.

5. Pan Z., Liu R., Zhang P., Zhou H., Fu Y., Zhou J. Combination of tigecycline and levofloxacin for successful treatment of nosocomial pneumonia caused by New Delhi Metallo- $\beta$-Lactamase-1-producing Raoultella planticola. Microb. Drug Resist., 2016, vol. 23, no. 1, pp. 127-131.

6. Sidjabat H.E., Townell N., Nimmo G. R., George N. M., Robson J., Vohra R., Davis L., Heney C., Paterson D. L. Dominance of IMP-4-producing Enterobacter cloacae among carbapenemase-producing Enterobacteriaceae in Australia. Antimicrob. Agents Chemother., 2015, vol. 59, no. 7, pp. 4059-4066. https://doi.org/10.1128/AAC. 04378-14.

7. Ahmad N., Ali S. M., Khan A. U. First reported New Delhi metallo- $\beta$-lactamase-1- producing Cedecea lapagei. In.t J. Antimicrob. Agents, 2017, vol. 49, no. 1, pp. 118-119. https://doi.org/10.1016/j.ijantimicag.2016.10.001.

8. Wang Q., Zhang Y., Yao X., Xian H., Liu Y., Li H., Chen H., Wang X., Wang R., Zhao C., Cao B., Wang $\mathrm{H}$. Risk factors and clinical outcomes for carbapenem-resistant Enterobacteriaceae nosocomial infections. Eur. J.Clin. Microbio.l Infect. Dis., 2016, vol. 35, pp. 1679-1689. https://doi.org/10.1007/ s10096-016-2710-0.

9. Quan J., Li X., Chen Y., Jiang Y., Zhou Z., Zhang H., Sun L., Ruan Z., Feng Y., Akova M., Yu Y. Prevalence of mcr-1 in Escherichia coli and Klebsiella pneumoniae recovered from bloodstream infections in China: a multicentre longitudinal study. Lancet Infect. Dis., 2017, vol.17, no. 4, pp. 400-410. https://doi. org/10.1016/S1473-3099(16)30528-X. 
10. Xie L., Dou Y., Zhou K., Chen Y., Han L., Guo X., Sun J. Coexistence of bla $a_{\mathrm{OX} A 48}$ and truncated bla $a_{\mathrm{NDM}-1}$ on different plasmids in a Klebsiella pneumoniae isolate in China. Front. Microbiol., 2017, vol. 8, p. 133. https://doi.org/10.3389/fmicb.2017.00133.

11. Joshi P. R., Acharya M., Kakshapati T., Leungtongkam U., Thummeepak R., Sitthisak S. Co-existence of bla $a_{\mathrm{OXA}-23}$ and bla $a_{\mathrm{NDM}-1}$ genes of Acinetobacter baumannii isolated from Nepal: antimicrobial resistance and clinical significance. Antimicrob. Resist. Infect. Control, 2017, vol. 6, pp. 1-7. https://doi. org/10.1186/s13756-017-0180-5.

12. Göttig S., Hamprecht A. G., Christ S., Kempf V. A., Wichelhaus T. A. Detection of NDM-7 in Germany, a new variant of the New Delhi metallo- $\beta$-lactamase with increased carbapenemase activity. J. Antimicrob. Chemother., 2013, vol. 68, no. 8, pp. 1737-1740. https://doi.org/10.1093/jac/dkt088.

13. Dortet L., Poirel L., Nordmann P. Worldwide dissemination of the NDM-type carbapenemases in Gram-negative bacteria. BioMed Res. Int., 2014, vol. 2014, Article ID 249856. https://doi. org/10.1155/2014/249856.

14. Gupta N., Limbago B. M., Patel J. B., Kallen A.J. Carbapenem-resistant Enterobacteriaceae: epidemiology and prevention. Clin. Infect. Dis., 2011, vol. 53, no. 1, pp. 60-67. https://doi.org/10.1093/cid/cir202.

15. Isozumi R., Yoshimatsu K., Yamashiro T., Hasebe F., Nguyen B.M., Ngo T.C., Yasuda S. P., Koma T.,

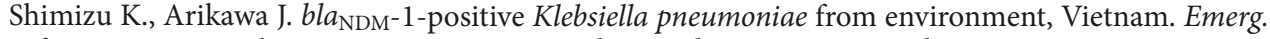
Infect. Dis., 2012, vol. 18, no. 8, pp. 1383-1385. https://doi.org/10.3201/eid1808.

16. Kim S. Y., Rhee J. Y., Shin S. Y., Ko K. S. Characteristics of communityonset NDM-1-producing Klebsiella pneumonia isolates. J. Med. Microbiol., 2014, vol. 63, no. 1, pp. 86-89. https://doi.org/10.1099/ jmm.0.067744-0.

17. Wailan A.M., Paterson D. L. The spread and acquisition of NDM-1: a multifactorial problem. Expert Rev. Anti. Infect. Ther., 2014, vol. 12, no. 1, pp. 91-115. https://doi.org/10.1586/14787210.2014.856756.

18. Shabanova V. V., Krasnova M. V., Bozhkova S. A., Ageevets V. A., Lazareva I. V., Rukina A. N., Sidorenko S. V. The first case of revealing of Klebsiella pneumoniae ST147, producing NDM-1 carbapenemase, in trauma and orthopedic hospital in Russia. Traumatology and Orthopedics of Russia, 2015, vol. 2, no. 76, pp. 90-98. (In Russian)

19. Suhorukova M.V., Jejdel'shtejn M.V., Skleenova E. J., Ivanchik N.V., Timokhova A.V., Dekhnich A.V., Kozlov R. S., Popov D. A. Antimicrobial resistance of nosocomial Enterobacteriaceae isolated in Russia: results of the national multicenter surveillance study "MARATHON" 2011-2012. Klinicheskaia mikrobiologiia $i$ antimikrobnaia khimioterapiia, 2014, vol. 16, no. 4, pp. 254-265. (In Russian)

20. Sidorenko S. V., Tishkov V. I. Molecular basis of resistance. Uspekhi biologicheskoi khimii, 2004, vol. 44, pp. 263-306. (In Russian)

21. Lev A.I., Astashkin E.I., Shaikhutdinova R.Z., Platonov M.E., Kartsev N.N., Volozhantsev N.V., Ershova O. N., Svetoch E.A., Fursova N. K. Identification of IS1R and IS10R elements inserted into ompk36 porin gene of two multidrug-resistant Klebsiella Pneumonia ehospital strains. FEMS Microbiol. Lett., 2017, vol. 364, no. 10, p. 364. https://doi.org/10.1093/femsle/fnx072.

22. Astashkin E. I., Lev A. I., Karcev N. N., Ershova O. N., Aleksandrova I. A., Svetoch E. A., Fursova N. K. Detection of the CTX-M-15 cephalosporinase gene and an atypical class 1 integron in a clinical isolate of Pseudomonas aeruginosa. Klinicheskaia mikrobiologiia $i$ antimikrobnaia khimioterapiia, 2016, vol. 18, no. 2, sup. 1, pp. 13-14. (In Russian)

Authors'information:

Olga V.Mironenko - MD, PhD (Medicine), Professor; miroolga@yandex.ru

Victoria V.Selnitseva - Doctor; selavik@mail.ru

Lidia A. Soprun — PhD (Medicine); lidas7@yandex.ru

Elena N.Shmushkevich - Doctor

Andrey A.Alekseev - Student; loafeeer@gmail.com

Aial S.Ivanov — Student; iv-aiaal@mail.ru

Anna A. Tovanova - Postgraduate Student; ann.tovan@bk.ru 